accuracy of such morphing, fairly precise endocranial volumes could be determined: $1,400 \mathrm{~cm}^{3}$ for DT, 1,440 for E and 1,325 for $\mathrm{RdM}$.

It is well known that Neanderthals have more robust cranial bones than modern humans, but it has been difficult to quantify this difference due to local fluctuation in bone thickness and variation in individual size. Computer-generated maps of the parietal bone (Fig. 1c) confirmed this fluctuation in thickness, but our capacity to sample at numerous points clearly revealed that average thickness of the parietal bone is elevated in Neanderthals relative both to parietal surface area and to cranial capacity (Fig. 2a,b). Similarly, Neanderthal mandibles are more robust in relation to the size of the braincase, and cross-sectional areas sampled along the dental arcade show distinctive profiles in Neanderthal and modern children (Fig. $2 c$ ).

In sum, using computer-assisted methods, marked quantitative differences in skull morphology can be demonstrated between modern humans and Neanderthals at an age of only 3-4 years. The existence of these clear quantitative differences at such an early age considerably strengthens the interpretation that Neanderthals and modern humans are separate species.

Christoph P.E. Zollikofer ${ }^{* \dagger}$

Marcia S. Ponce de León*

Robert D. Martin ${ }^{*}$, Peter Stucki ${ }^{\dagger}$

*Anthropologisches Institut und Museum

and ${ }^{\dagger}$ MultiMedia Laboratorium,

Institut für Informatik, Universität Zürich,

CH-8057 Zürich, Switzerland

\section{Discontinuance of SMOW and PDB}

SIR - Confusion exists in the reporting of stable hydrogen, carbon and oxygen isotope results, because the supply of the PDB (Peedee belemnite) standard has been exhausted and because SMOW (standard mean ocean water) does not have a unique definition. Because laboratories do not use the same reference material to establish their isotope-ratio scales, different laboratories report widely varying values for the same material.

To eliminate confusion in the reporting of light stable isotope ratios, the Commission on Atomic Weights and Isotopic Abundances of the International Union of Pure and Applied Chemistry has recommended ${ }^{1,2}$ that the use of SMOW and PDB be discontinued and that isotope abundances of hydrogen-, carbon- and oxygenbearing materials be reported relative to the reference water VSMOW (Vienna standard mean ocean water) and to VPDB (Vienna Peedee belemnite), defined by adopting a $\delta^{13} \mathrm{C}$ value of $+1.95 \%$ and a $\delta^{18} \mathrm{O}$ value of $-2.20 \%$ for NBS 19 carbonate relative to
VPDB. The isotope abundance scales should be normalized so that SLAP (standard light Antarctic precipitation) has a $\delta^{2} \mathrm{H}$ and $\delta^{18} \mathrm{O}$ of -428 and $-55.5 \%$, respectively, relative to VSMOW. If the isotope abundance measurement of a sample depends on an isotopic fractionation factor, this should be reported.

\section{Tyler B. Coplen}

Commission on Atomic Weights and

Isotopic Abundances,

International Union of Pure and Applied

Chemistry,

US Geological Survey,

Reston, Virginia 22092, USA

1. IUPAC Pure appl. Chem. 66, 2423-2444 (1994)

2. Coplen, T.B. Pure appl. Chem. 66, 273-276 (1994).

\section{Apolipoprotein E and Alzheimer's}

SIR - The report by Games and colleagues ${ }^{1}$ of the first successful generation of transgenic mice overexpressing pathological human $\beta$-amyloid precursor protein (APP) which also exhibit some of the pathological features of Alzheimer's disease is undoubtedly a milestone. Nevertheless, it may not yet be possible to conclude, as did Duff and Hardy in their News and Views article ${ }^{2}$, that this result settles the argument for the primacy of amyloid deposition in Alzheimer's disease neuropathology as opposed to other factors such as apolipoprotein E4 (apo E4) ${ }^{3}$.

Although cysteine-containing apo E3 is by far the most frequently occurring isoform in humans, apo E4 is considered the ancestral form ${ }^{4}$. In most species, including mouse, apo E lacks cysteine and is E4-like, with arginine occupying the equivalent of amino-acid positions 112 and 158 (ref. 5). The findings by Games and colleagues cannot, therefore, exclude a role for apo E4 in the pathogenesis of Alzheimer's disease, as the mice in their study are by one definition homozygous for apo E4.

Indeed, their results could be taken further to implicate a role for apo E4 in the disease process. To exclude such a role for E4, the analyses should be repeated in the progeny from matings of their mice with those lacking apo $\mathrm{E}$. It is interesting that transgenic mice lacking apo $\mathrm{E}$ and people with apo $\mathrm{E}$ deficiency appear neurologically normal ${ }^{6,7}$.

Frank M. van Bockxmeer

Department of Biochemistry,

Royal Perth Hospital, Perth,

Western Australia 6000, Australia

Games, D. et al. Nature 373, 523-527 (1995)

2. Duff, K. \& Hardy, J. Nature 373, 476-477 (1995).

3. Hyman, B. T. et al. J. Neuropath. exp. Neurol. 53, $427-428$ (1994).

4. Weisgraber, K. H. Adv. Protein Chem. 45, 249-302 (1994).

5. Chan, L. \& LI, W.-H. Curr. Opin. Lipidol. 2, 96-103 (1991)

6. Popko, B. et al. J. Neurochem. 60, 1155-1158 (1993).

7. Lohse, P. et al. J. Lipid Res. 33, 1583-1590 (1992).
Hypermutation in $T$ cells questioned

SIR - Zheng et al. ${ }^{1}$ investigated the occurrence of point mutations in rearranged T-cell antigen receptor genes in different compartments of the spleen of immunized mice. Because of the antigen used, the immune response is clonally restricted, mostly to $\mathrm{V} \beta 3$ and $\mathrm{V} \alpha 11$ genes. The authors have amplified a subset of rearranged $T$-cell receptor genes from pools of 20-40 cells from the periarteriolar T-cell sheath (PALS) and germinal centres (GCs), then subcloned and sequenced $\mathrm{V}(\mathrm{D}) \mathrm{J}$ gene segments.

The authors found that the error frequency of $14.2 \times 10^{-4}$ per base pair for Vo11 segments recovered from GCs was much higher than for those recovered from the PALS $\left(2.1 \times 10^{-4}\right)$ or a T-cell line $\left(0.7 \times 10^{-4}\right)$. We would like to point out that even these large differences are not necessarily significant in replicating systems, which are intrinsically subject to large fluctuations. This is the fluctuation effect that Luria and Delbrück ${ }^{2}$ used more than 50 years ago to prove that streptomycin-resistant bacteria were due to preexisting mutations in the culture rather than to variants induced by the drug. Unfortunately, Zheng et al. ${ }^{1}$ provide insufficient data for a proper quantitative analysis, and it is unlikely that the experiments were designed with that in mind.

The authors clearly believe that the mutations pre-existed PCR amplification. They say that a bias for mutations in nonproductive alleles is indicative of counterselection against mutants and thus inconsistent with "abiotic" processes. Again, the data provided do not allow one to decide whether there is selection against mutations, or no selection and merely fluctuation.

The successive accumulation of point mutations was first recognized by Weigert et $a l^{3}$ to be the hallmark of affinity maturation plus hypermutation at immunoglobulin loci. Only in one clonally related family do Zheng et al. ${ }^{1}$ find a successive accumulation of point mutations. Most unique mutations are found in this family. It is only a single clone, but we find this result to be much more suggestive than the claim of a quantitative difference between the GC and the PALS.

There is one problem. How do we know that the mutations arose in T cells? An equally tenable hypothesis would be that some GC B cells rearrange and hypermutate T-cell receptor genes. The authors cannot invoke the widely accept-

1. Zheng, B., Xue, W. \& Kelsoe, G. Nature 372, 556-559 (1994)

2. Luria, S. \& Delbrück, M. Genetics 28, 491-511 (1943).

3. Weigert, M., Cesari, I.M., Yonkovich, S.J. \& Cohn, M. Nature 228, 1045-1047 (1970). 